\section{Video tracking analysis of behavioral scores: The Finnover experience}

\author{
Hanna Trebesova, ${ }^{1}$ Federica Turrini, ${ }^{1}$ \\ Guendalina Olivero, 1 \\ Valentina Orlandi, ${ }^{1}$ Dario Donno, ${ }^{2}$ \\ Gabriele Beccaro, ${ }^{2}$ Raffaella Boggia, \\ Anna Pittaluga, ${ }^{1}$ Massimo Grilli ${ }^{1}$
}

1Department of Pharmacy, University of Genoa; 2Department of Agriculture, Forestry and Food Science, University of Torino, Turin, Italy

\begin{abstract}
The aim of this study was to reduce and refine the number of animals subjected to behavioral analysis by ToxTrac software during the European project InterregALCOTRA "Finnover".
\end{abstract}

\section{Introduction}

Open-source software development gives the possibility to refine the experimental design involving animal models. ${ }^{1}$ Video tracking is particularly suitable for mice behavioral analysis. Accordingly, in this study ToxTrac tracking software, an open-source software by Rodriquez, ${ }^{2}$ was used. ToxTrac allows the acquisition of more data, eliminates the subjective influence and reproducibility problems, and at last, is less time consuming. ${ }^{3}$ Interestingly, the system provides locomotor information such as average speed, mobility rate, exploration rate and total distance. In several mice behavioral assays this information could be linked to a specific movement (grooming, rearing etc.) or behavior traits (sociality, aggression, exploration etc.). ${ }^{2}$ Our results show the application of the video tracking analysis during the European project Interreg-ALCOTRA "Finnover" to study the effects of two different Tilia Tomentosa extracts on mice.

\section{Materials and Methods}

Animals: young (3-6 months) and old (20-24 months) male mice C57/BL.

Dietary supplementation: TTBEs (Tilia tomentosa bud extracts) and UTTBEs (Ultrasound Tilia tomentosa bud extracts) were dissolved in drinking water $(500 \mu \mathrm{L}$ in $500 \mathrm{~mL}$ ) and administered for 21 days. ${ }^{4}$
Behavioral analysis: Hole board: Each animal was placed singly in the center of the board and its behavior recorded with a video camera for 5 min. Light/dark and Elevated Plus Maze: Each mouse was placed in the center of the arena, and then the operator started to record 5 min of spontaneous exploration. Marble test: The test was performed using a squared arena containing a $5 \mathrm{~cm}$ high sawdust layer. Twenty glass marbles beads were placed before the test phase and a 10-minute video recording was performed.

Animals were recorded on day 0 (t0) and on day 21 ( $\mathrm{t} 21)$. Both $\mathrm{t} 0$ and $\mathrm{t} 21$ video were analyzed by ToxTrac and directly by three different researchers. Subsequently, the data obtained were analyzed trough univariate and multivariate statistical analysis.

ToxTrac: https://toxtrac.sourceforge.io

\section{Results}

The data allow us to discriminate in a precise manner the difference between young and old mice from a behavioral and motor point of view. The robust statistical data demonstrate a significant anxiolytic effect in young mice rather than in old mice, which is less evident. The use of video tracking software increased the number of variables investigated allowing to reduce the experiments needed.

\section{Discussion and Conclusions}

From a 3R perspective, the coupling of video tracking with statistical analysis
Correspondence: Massimo Grilli, Department of Pharmacy, University of Genoa, Italy.

E-mail: grilli@difar.unige.it

Key words: Video tracking analysis; behavior; natural compounds.

Acknowledgments: This research was funded by a European Union project called FINNOVER ( $\left.n^{\circ} 1198\right)$, http://www.interregfinnover.com/.

Disclosures: Authors have nothing to disclose

Conference presentation: This paper was presented at the Third Centro 3R Annual Meeting - L'era delle 3R: modelli in silico, in vitro e in vivo per promuovere la ricerca traslazionale 30 September - 1 October 2021, Evento online organizzato dal Politecnico di Torino.

Received for publication: 9 July 2021.

Accepted for publication: 7 September 2021.

This work is licensed under a Creative Commons Attribution NonCommercial 4.0 License (CC BY-NC 4.0).

CCopyright: the Author(s), 2021

Licensee PAGEPress, Italy

Biomedical Science and Engineering 2021; 4(s1):197 doi:10.4081/bse.2021.197

allowed optimizing the experimental design and reduce the number of animals. Interestingly, from a pharmacological point of view, the software analysis revealed therapeutic properties of TTBEs and UTTBEs that could be utilized against animal mood disorders.
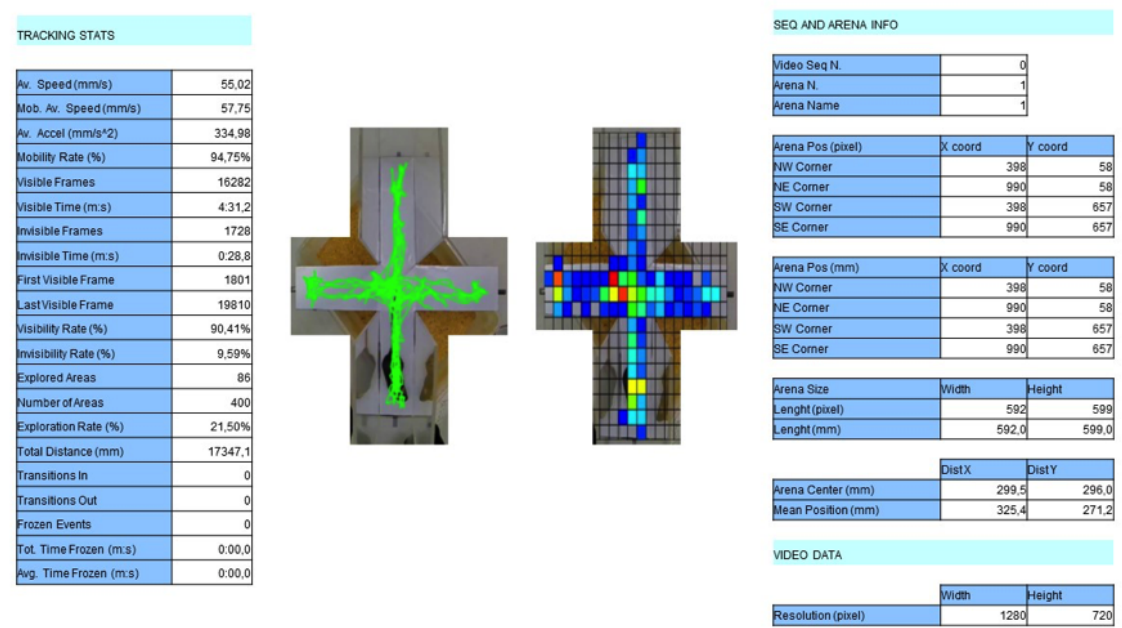

Figure 1. Representative image of ToxTrac analysis 
for tracking organisms. Methods Ecol Evol 2018;9:460-4.

References

1. von Krogh $\mathrm{G}$, von Hippel E. The promise of research on open source software. Manag Sci 2006;52:975-83.

2. Rodriguez A, Zhang H, Klaminder J, et al. ToxTrac: a fast and robust software Barros KM, et al. Automatic system for analysis of locomotor activity in rodents - a reproducibility study. J Neurosci Methods 2011;195:216-21.
3. Aragão Rda S, Rodrigues MA, de
4. Turrini F, Vallarino G, Cisani F, et al. Use of an animal model to evaluate anxiolytic effects of dietary supplementation with Tilia tomentosa moench bud extracts. Nutrients 2020;12:3328. 